\title{
Fundamental solitons in discrete lattices with a delayed nonlinear response
}

\author{
A. Maluckov ${ }^{1}$, Lj. Hadžievski ${ }^{2}$, and B. A. Malomed ${ }^{3}$ \\ 1 Faculty of Sciences and Mathematics, University of Niš, P.O. Box 224, 18001 Niš, Serbia \\ 2 Vinča Institute of Nuclear Sciences, P.O. Box 522,11001 Belgrade, Serbia \\ 3 Department of Physical Electronics, School of Electrical Engineering, \\ Faculty of Engineering, Tel Aviv University, Tel Aviv 69978, Israel
}

\begin{abstract}
The formation of unstaggered localized modes in dynamical lattices can be supported by the interplay of discreteness and nonlinearity with a finite relaxation time. In rapidly responding nonlinear media, on-site discrete solitons are stable, and their broad inter-site counterparts are marginally stable, featuring a virtually vanishing real instability eigenvalue. The solitons become unstable in the case of the slowly relaxing nonlinearity. The character of the instability alters with the increase of the delay time, which leads to a change in the dynamics of unstable discrete solitons. They form robust localized breathers in rapidly relaxing media, and decay into oscillatory diffractive pattern in the lattices with a slow nonlinear response. Marginally stable solitons can freely move across the lattice.

PACS numbers: 05.45.-a; 05.45.Yv
\end{abstract}

The delay of the medium's response is unavoidable in natural phenomena and technology. In this respect, it is relevant to mention the cellular physiology, genetics, signal processing, transport of impulses through neural networks, the propagation of light signals in optical resonator systems and cavities, and so on. The delayed response may significantly affect many phenomena in these fields. In particular, essential issues, which are addressed in this paper, are the existence, stability and dynamics of localized modes in dynamical lattices with the non-instantaneous nonlinear response. The lattice, which may describe an array of nonlinear optical waveguides, is modeled by the timedelayed discrete nonlinear Schrödinger equation. The localized modes in this model are explored using a properly modified delay-differential equation solver package DDE-BIFTOOL v. 2.00. We show that the discrete bright on-site and inter-site solitons (so classified according to the location of their center) can be created by the modulational instability of continuous waves, which occurs in the same parameter region as in the lattice with the instantaneous nonlinearity. However, the stability of the solitons is affected by the delayed response in three different ways: (i) the solitons are destroyed when the delay time exceeds a certain critical value; (ii) in the case of the fast nonlinear response (short temporal delay), the instability growth rate of inter-site solitons decreases in comparison with the instantaneous model; (iii) the instability type changes for intermediate values of the delay time. The inter-site and on-site solitons with very close values of the power in the fast-responding media evolve into localized breathing modes when their amplitude is slightly perturbed. They can also be transformed into moving localized modes by the application of a kick. However, larger values of the delay time enhance the temporal correlation between time-distant events, which results in bringing moving solitons to a halt.

\section{INTRODUCTION}

Usually, whenever an irregular, unpredictable change occurs in an engineering device, the need arises to take action in order to make the perturbation predictable or suppress it altogether. An solution of this problem is based on a delayed feedback, in the form of a signal proportional to the difference between the current value of some parameter of the system's state and its value in the past. Taking the time delay equal to the period of the unwanted random oscillations, the evolution may be made stable, with the appropriate choice of the feedback strength [1 3$]$.

In particular, systems built as recurrent neural networks have found many applications to signal and image processing, pattern recognition, realization of associative memories, solving certain optimization problems, and in other fields. Because delays of integration and communication are unavoidable, often causing an instability of the systems, attention has been focused on the stability of neural networks with a temporal delay [4 [6]. Time-delayed systems also play a significant role in modeling living cells, genetics, etc. 7 [-9].

In the field of optics, a strong delay is demonstrated by coupled-resonator optical waveguides (CROWs), which are built as side-coupled densely packed chains of spherical cavities [10]. It may happen that a light wave spends much more time circulating in each cavity than hopping between them, which makes the group velocity of the light transmission very low. Because the time delay induced by the sojourn of the light signal in the resonator strongly depends on 
the detuning of the signal from the resonator's eigenfrequency, CROWs feature the group-velocity dispersion which is much stronger than in conventional materials [11, 12]. Applications suggested by such settings include various slow-light phenomena, transmission lines with a variable optical delay, clean pulse compression on a chip without formation of a pedestal, etc. [13-17].

The above-mentioned examples demonstrate that the delay of the medium's response may significantly affect various fundamental phenomena and applications. In this regard, an essential issue is the dynamics of localized modes in media with the non-instantaneous nonlinear response. In particular, it was demonstrated that the relaxation of the nonlinearity has a strong influence on the wave-packet dynamics [17, 18].

The objective of the present work is to study the existence, stability and dynamics of localized modes in onedimensional discrete lattices with the on-site non-instantaneous nonlinear response. We find that the solitons in this case can be formed by the modulation instability (MI), similarly to the lattice with the instantaneous response. We explore the dependence of the stability and dynamical properties of fundamental unstaggered localized modes in the lattice on the response time ("unstaggered" means that the sign of the lattice field does not jump between adjacent sites [19]).

It is relevant to mention that the stability and related dynamical properties of the time-delayed systems have been studied in some detail since the pioneering work [20], where several stability criteria for discrete delayed systems were derived, in a form independent of the value of the delay time. A rigorous stability analysis has been performed for simple linear and nonlinear systems with the temporal delay, that were modeled by differential-difference equations [21, 22], primarily with the objective to predict a possibility of controlling the system through the time-delayed feedback. In addition, the stability and bifurcations of fixed points have been numerically investigated for a few simple nonlinear models of the neural activity [23]. In this work, we looked for numerical solutions for the stationary solitons in the TD-DNLSE by means of modified dde-solver tools 27], while the stability was analyzed using a properly modified MATLAB program package DDE-BIFTOOL v. 2.00 [23, 28].

The rest of the paper is structured as follows. The model is formulated in Section II, following Ref. [18], where it was introduced for electron-photon systems featuring a nonadiabatic interaction. The MI is also considered in Section II. The main results concerning the stability and dynamical behavior of the discrete solitons are presented in Section III. The paper is concluded by Section IV.

\section{THE MODULATION INSTABILITY IN LATTICES WITH THE NON-INSTANTANEOUS NONLINEARITY}

In solid-state media, the transmission of electron wave packets through chains with a nonadiabatic on-site electronphonon interaction may be modeled by the time-delayed discrete nonlinear Schrödinger equation (TD-DNLSE), which was proposed, in this context, in Ref. [18]:

$$
i \frac{d \psi_{n}(t)}{d t}+C\left[\psi_{n+1}(t)+\psi_{n-1}(t)-2 \psi_{n}(t)\right]-\kappa\left|\psi_{n}(t-\tau)\right|^{2} \psi_{n}(t)=0,
$$

where $n$ is the discrete coordinate, $\tau$ is the delay time, $C>0$ is the coupling constant, and $\kappa$ is the nonlinearity parameter which determines the sign of the nonlinearity, $\kappa=-1$ and +1 corresponding to the self-attraction and selfrepulsion, respectively. In the simplest approximation, this model may be applied to the description, in the temporal domain, of optical systems in the form of the above-mentioned CROWs. In the next section, we focus on the case of $\kappa=-1$, when the unstaggered solitons exist in the model with the instantaneous nonlinearity.

The only dynamical invariant which remains conserved in the presence of the non-instantaneous nonlinearity is the total norm (alias energy, in terms of the CROW-like optical models):

$$
P(t)=\sum_{n=1}^{N}\left|\psi_{n}(t)\right|^{2} .
$$

Below, it will be used as the main characteristic of localized modes.

As is well known [19, 24, 25], the instantaneous DNLSE exhibits the MI of continuous-wave (CW) states in the lattice, which is a mechanism for the creation of discrete solitons. The MI is a result of the competition between the discreteness and the nonlinearity. Here we analyze how the delayed response affects the MI.

Equation (11) gives rise to the $\mathrm{CW}$ solution, $\psi_{n}(t)=A \exp (-i \mu t)$, with frequency $\mu$ and constant amplitude $A=\sqrt{\mu / \kappa}$, in the regions of $\mu<0$ for $\kappa=-1$, and $\mu>0$ for $\kappa=+1$. The MI can be investigated, in the framework of the linear-stability analysis, by adding a small complex perturbation to the CW solution,

$$
\psi_{n}(t)=\left[A+\delta u_{n}(t)\right] \exp (-i \mu t)
$$




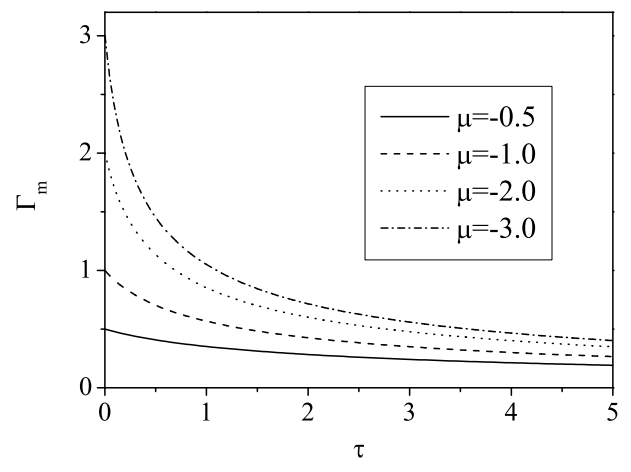

FIG. 1: The MI growth rate, $\Gamma_{m}$, corresponding to the fastest growing modulation mode, versus the delay time, $\tau$, for a few fixed values of frequency $\mu$ which determines the amplitude of the unstable CW state (3).

[19, 24, 25]. Looking for solutions for the perturbations in the form of $\delta u_{n}(t) \sim \exp (i q n) \exp (\Gamma t)$, where $q$ is the wavenumber and $\operatorname{Re}\{\Gamma\}$ is the growth rate of the perturbation, we obtain the dispersion relation for the lattice with the delayed response, in the form of the following transcendental equation:

$$
\Gamma^{2}=-8 C \sin ^{2}(q / 2)\left[2 C \sin ^{2}(q / 2)+\mu \exp (-\Gamma \tau)\right] .
$$

The CW solution is unstable if Eq. (44) gives rise, for some wavenumbers $q$, to real positive $\Gamma$. Obviously, this is possible for $\mu<0$, which coincides with the MI condition at $\tau=0$ (recall the $\mathrm{CW}$ with $\mu<0$ exists for $\kappa=-1$, i.e., in the case of the self-attractive nonlinearity). However, the time delay affects the magnitude of $\Gamma$. The expression for the growth rate corresponding to the fastest-growing instability mode can be obtained from Eq. (4):

$$
\Gamma_{m}=W(-\mu \tau) / \tau
$$

where $W(x)$ is the Lambert's $W$ function [26], defined as a solution to equation $x=W e^{W}$. The dependence of $\Gamma_{m}$ on $\tau$, which was numerically calculated for different values of $\mu$, is presented in Fig. 1. It clearly shows that the increase of the delay time leads to a decrease of $\Gamma_{m}$.

\section{THE STABILITY AND DYNAMICS OF FUNDAMENTAL SOLITONS}

\section{A. The general approach}

The development of the MI may split the CW into an array of solitons. The stationary solution for a discrete soliton with frequency $\mu$ can be looked for as $\psi_{n}(t)=\phi_{n} \exp (-i \mu t)$, where $\phi_{n}$ is a real lattice field, subject to conditions $\phi_{n \rightarrow \pm \infty} \rightarrow 0$, which satisfies the following equation:

$$
\mu \phi_{n}+C\left(\phi_{n+1}+\phi_{n-1}-2 \phi_{n}\right)+\phi_{n}{ }^{3}=0
$$

where we set $\kappa=-1$, as said above, because unstaggered solitons do not exist in the opposite case. Equation (6) has the same form as in the case of $\tau=0$, hence the shape of the stationary solitons (unlike their stability) is independent on the non-instantaneous character of the nonlinearity. For this reason, examples of stationary discrete solitons are not displayed here (they are identical to those in the standard DNLSE with the instantaneous self-attractive nonlinearity [19]).

The stability of the solitons was analyzed following the standard linearization procedure. To this end, a small complex perturbation is added to the soliton,

$$
\psi_{n}(t)=\left[\phi_{n}+\delta a_{n}(t)+i \delta b_{n}(t)\right] \exp (-i \mu t),
$$

where $\delta a_{n}$ and $\delta b_{n}$ are real functions, and $\mu$ is the same frequency of the unperturbed soliton which was defined above. Substituting expression (7) into Eq. (11) and performing the linearization, a system of equations for the small 

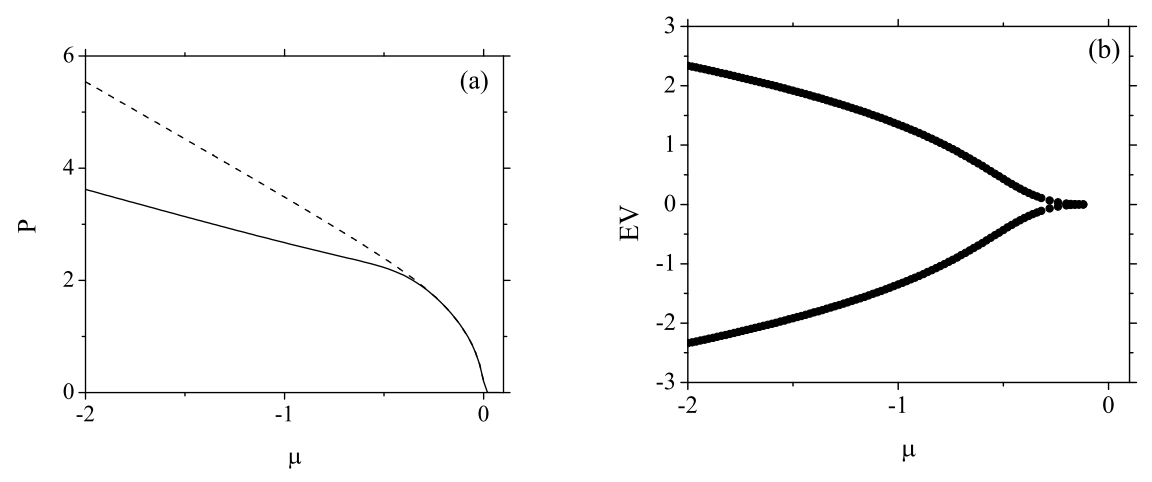

FIG. 2: (a) Norm versus $\mu$ for on-site (solid line) and inter-site (dashed line) stationary fundamental solitons in the lattice with $C=0.8$. The EV (eigenvalue) spectrum for the inter-site soliton in the absence of the temporal delay, $\tau=0$, includes a pair of pure real EVs, as shown in (b), for all $\mu$, except in an area close to $\mu=0$, where the unstable EV practically vanishes, and the on-site counterpart of the inter-site soliton, with the same norm, can be found, cf. panel (a).

perturbations is obtained:

$$
\begin{aligned}
\frac{d}{d t} \delta a_{n}(t) & =\left[(2 C-\mu)-\phi_{n}^{2}\right] \delta b_{n}(t)-C\left[\delta b_{n+1}(t)+\delta b_{n-1}(t)\right], \\
\frac{d}{d t} \delta b_{n}(t) & =-\left[(2 C-\mu)-\phi_{n}^{2}\right] \delta a_{n}(t)+C\left(\delta a_{n+1}(t)+\delta a_{n-1}(t)\right)+2 \phi_{n}^{2} \delta a_{n}(t-\tau),
\end{aligned}
$$

which can be rewritten in the matrix form:

$$
\frac{d}{d t} U(t)=M_{1} U(t)+M_{2} U(t-\tau)
$$

$U(t)$ being the column transpose to $\left\{\delta a_{1}(t) \ldots \delta a_{N}(t) \delta b_{1}(t) \ldots \delta b_{N}(t)\right\}$, and $M_{1,2}$ are the corresponding $2 N \times 2 N$ matrices. A solution of Eq. (10) gives rise to $2 N$ stability eigenvalues (EVs), the soliton being unstable if there are EVs with nonzero real parts. The instability is exponential or oscillatory if, respectively, the corresponding EVs are real or complex. In the area-preserving models (Hamiltonian lattices, which is the case only for $\tau=0$ ), real EVs always appear in pairs of positive and negative ones with equal absolute values, while complex EVs appear in quartets featuring different combinations of the signs in the front of the real and imaginary parts [19]. Obviously, the presence of the time delay in Eq. (10) may alter the EVs and affect the stability of the discrete solitons.

Our main aim is to study the stability of stationary unstaggered fundamental solitons in the lattice system with the finite response time. We consider the lattice subject to periodic boundary conditions, with the soliton's center placed either at the site of the lattice (on-site solitons), or between two sites (inter-site solitons). Results presented below were obtained for the lattices with $N=100$ or 101 sites, for the inter-site and on-site modes, respectively. Values of the delay time were taken in the interval of $0<\tau<5$.

The lattices with the instantaneous nonlinear response support unstaggered fundamental solitons of the two abovementioned types, on-site and inter-site ones [19, 24, 25]. In terms of the corresponding EVs of small perturbations, the on-site solitons are stable in the Kerr media with the attractive cubic nonlinearity, while the inter-site solitons are unstable. A specific situation occurs at $\mu \rightarrow-0$, where the unstable inter-site solitons can find on-site counterparts with very close values of the norm (2), see Fig. 2(a). In that case, the solitons of both types may be called marginally stable 25], as the instability growth rate of the inter-site ones virtually vanishes, see Fig. 2(b), and the dynamical behavior of the on-site and inter-site solitons with equal norms is nearly identical (under the action of perturbations, they become breathers, see below). These solitons feature large widths and small amplitudes.

Being slightly perturbed, the unstable inter-site soliton spontaneously shifts by half a lattice spacing, to transform itself into a stable on-site counterpart with the same norm. In the region of the coexistence of inter-site and onsite solitons with nearly equal norms, a kicked soliton may move along the lattice, by hopping between the on-site and inter-site configurations, overcoming the Peierls-Nabarro potential barrier. Through this mechanism, the barrier effectively vanishes for the marginally stable solitons, making them fully mobile objects. 


\section{B. Inter-site solitons}

Marginally stable inter-site solitons, which we again define as those with virtually vanishing real eigenvalues, and with on-site counterparts possessing the same norm, can be found, at relatively small values of $|\mu|$, in the lattice with a sufficiently short delay of the nonlinear response, as shown in Fig. 3(a) for $\mu=-0.22$ and $C=0.8$. All the values of the instability growth rate in Fig. 3(a) are bounded to $\operatorname{Re}(\mathrm{EV}) \lesssim 0.001$.
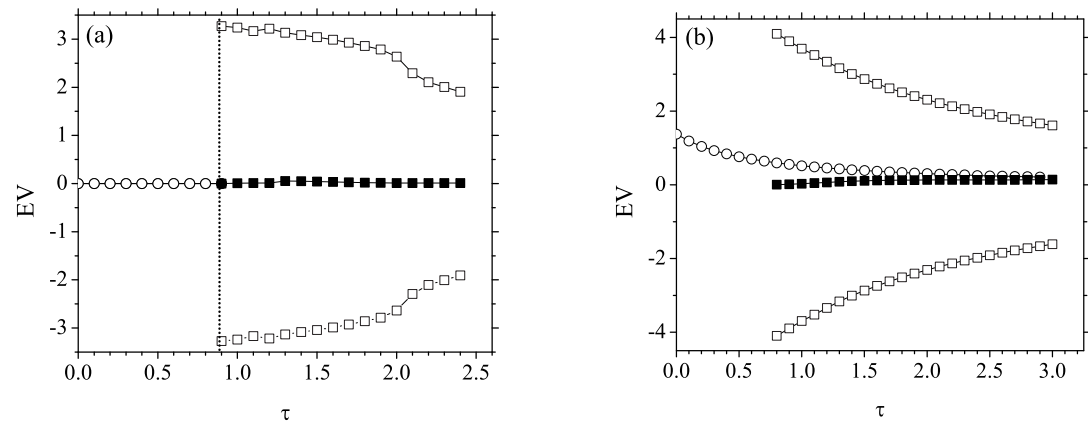

FIG. 3: The EVs (eigenvalues) with the largest real part are shown, as functions of the delay time, $\tau$, for inter-site solitons: (a) at $\mu=-0.22$, and (b) at $\mu=-1.02$. The lattice coupling constant is $C=0.8$. Dark and white squares denote real and imaginary parts of the complex EVs. In panel (a), the dotted line separates the region of the marginally stable inter-site solitons from that where unstable complex EVs emerge. In panel (b), the dotted line separates an area of the exponential instability (left region - circles) from that with many complex EVs. Only complex EVs with the largest real part are plotted (showing the entire set of the EVs would make the plots messy). In (b), the pure real EV and the real part of the complex EV become comparable at $\tau>1.5$.
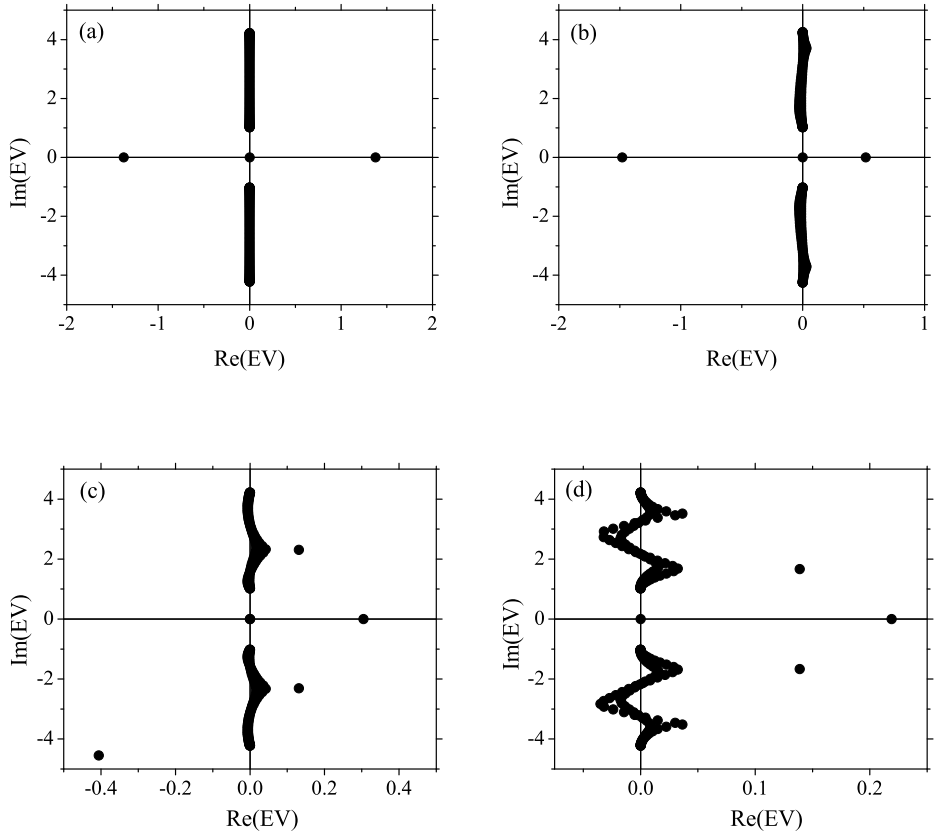

FIG. 4: The EV spectrum, shown in the form of the EV's imaginary part versus the real part, for inter-site solitons. Parameters are $C=0.8, \mu=-1.02$, and $\tau=0(\mathrm{a}), \tau=1(\mathrm{~b}), \tau=2(\mathrm{c})$, and $\tau=3(\mathrm{~d})$. All these values correspond to particular points in Fig. 3(b). In the case of $\tau=0$, the corresponding inter-site soliton is exponentially unstable [see Fig. 3(b)]. With the increase of $\tau$, complex EVs with a significant real part start to emerge, although the real EV remains the dominating one. 

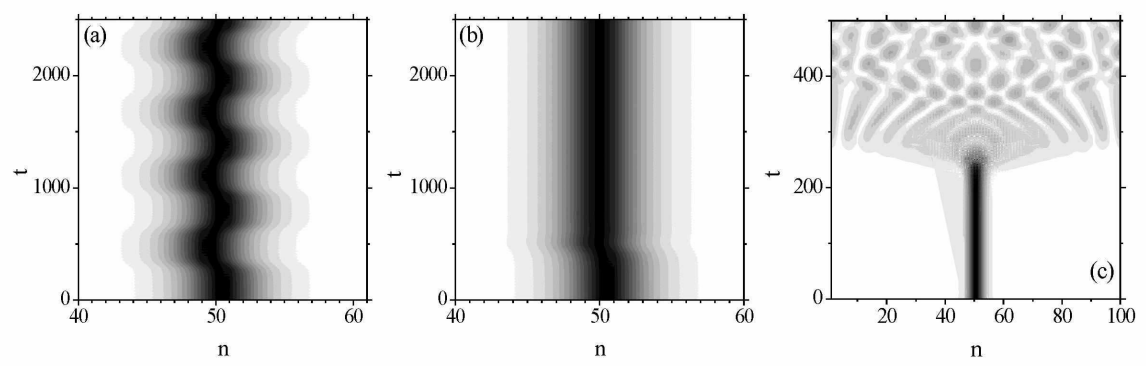

FIG. 5: The evolution of a perturbed inter-site soliton with $C=0.8, \mu=-0.22$. For small $\tau$, it is marginally stable. Being slightly perturbed, it evolves into a breather at $\tau=0$ (a), or into an on-site-centered soliton at $\tau=0.2$ (b). At larger values of the delay time $\tau$, the inter-site soliton is strongly unstable, decaying into a diffractive pattern at $\tau=1.2(\mathrm{c})$.

The instability of the inter-site solitons becomes stronger at larger values of $|\mu|$ (for more narrow solitons). The unstable solitons develop two types of instability in the lattice with the non-instantaneous nonlinear response. In the case of small and medium values of the delay time, $\tau$, the instability growth rate, represented by the dominant pure real eigenvalue in Fig. 3(b), is smaller than in the case of the instantaneous nonlinearity. However, the inter-site solitons do not become truly stable in this case (barring the above-mentioned marginally stable one found at smaller values of $|\mu|$ ). As seen in Figs. 3 (b) and 4 the increase of $\tau$ leads to the creation (starting at $\tau=0.9$ ) of a growing number of complex eigenvalues with positive real parts that account for additional oscillatory instabilities, although the real eigenvalue remains the largest one.

In the TD-DNLSE, as well as in the DNLSE with the instantaneous nonlinearity, the long-time evolution of unstable solitons depends on the initial perturbation. In the case of zero or very small $\tau$, adding a small-amplitude perturbation to the inter-site mode belonging to an inter-site/on-site soliton pair with equal norms (i.e., it is a marginally stable soliton, as defined above), gives rise to a breathing mode, see Fig. 5(a). On the contrary, at larger values of $\tau$ (when the inter-site soliton is no longer marginally stable, being explicitly unstable), an on-site-centered localized mode appears as a result of the instability development [Fig 5(b)]. In the model with still larger $\tau$ (in the slowly responding medium), the same unstable inter-site mode suffers an instability-induced diffraction into an oscillating pattern, as shown in Fig. [5(c). Diffractive patterns are also a final state of the evolution of slightly perturbed unstable inter-site modes which do not have on-site (equal-norm) counterparts.

\section{On-site solitons}

On-site solitons, which remain stable at sufficiently small values of the delay time $\tau$, get destabilized with the increase of $\tau$. This is indicated by a slowly growing pure real EV, corresponding to an eigenmode of small perturbations which accounts for sharpening of the perturbed on-site soliton, and by the emergence of a growing number of complex EVs with positive real parts. For instance, the destabilization of the on-site solitons is observed at $\tau>0.7$ for $\mu=-1.02$ and $C=0.8$, as shown in Fig. 6. The perturbed on-site soliton in the slowly responding media (large $\tau$ ) decays into a diffractive oscillatory pattern due to the excitation of unstable oscillatory modes, cf. Fig. 5(c) which shows a similar outcome of the instability development of the inter-site solitons.

The critical value of $\tau$ at which the instability of the on-site solitons sets in varies as a function of the soliton's norm, as shown in Fig. 7 The delay effects, including the corresponding instability, stronger affect tightly localized modes with a large norm, whose behavior is dominated by the nonlinearity, then their broad small-amplitude counterparts. However, it is necessary to mention that tightly pinned modes (essentially, those sitting on a single lattice site) may be actually unphysical, if the underlying continual model, approximated by the discrete one, admits the collapse, as in the case of the one-dimensional nonpolynomial Schrödinger equation which describes the Bose-Einstein condensate with the intrinsic attraction loaded into a deep optical-lattice potential 29].

Local modes which are members of marginally stable inter-site and on-site soliton pairs with equal norms, which exist in the case of small $\tau$, can be set in motion across the lattice by the application of the kick, i.e., taking $\psi_{n}(t=0)=\phi_{n} \exp (i \alpha n)$, similar to the behavior of the marginally stable solitons in the lattice with the instantaneous nonlinearity. Therefore, the concept of the effective Peierls-Nabarro barrier can be applied to the lattices with the time-delayed nonlinearity. However, larger values of $\tau$ enhance the temporal correlation between time-distant events, favoring bringing moving solitons to a halt, as shown in Fig. 8 . 

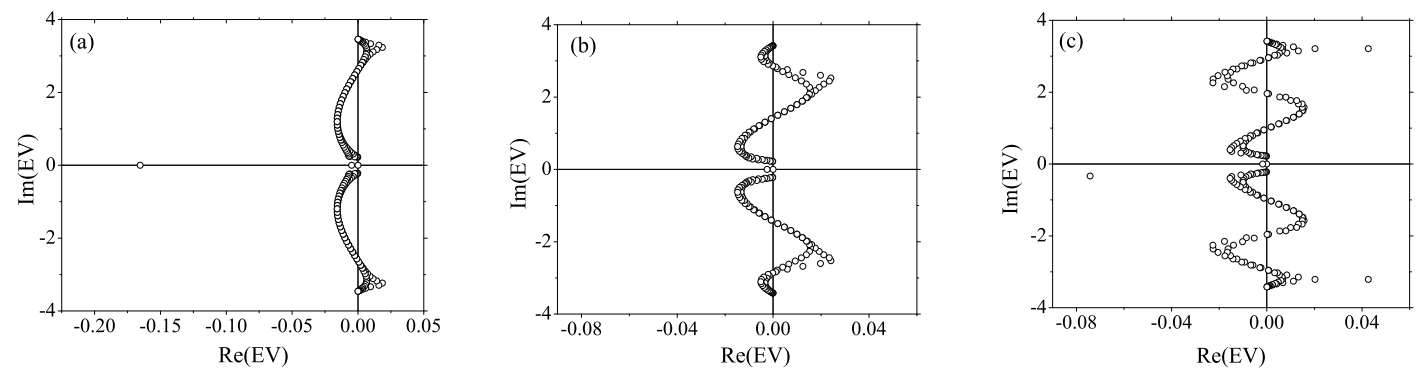

FIG. 6: The EV spectra for unstable on-site solitons in media with the non-instantaneous nonlinear response, cf. Fig. 4 for inter-site solitons. Parameter values are $C=0.8, \mu=-1.02$, and $\tau=1$ (a), $\tau=2(\mathrm{~b}), \tau=3$ (c). In the case of a small delay time $\tau$, the corresponding on-site soliton is stable.

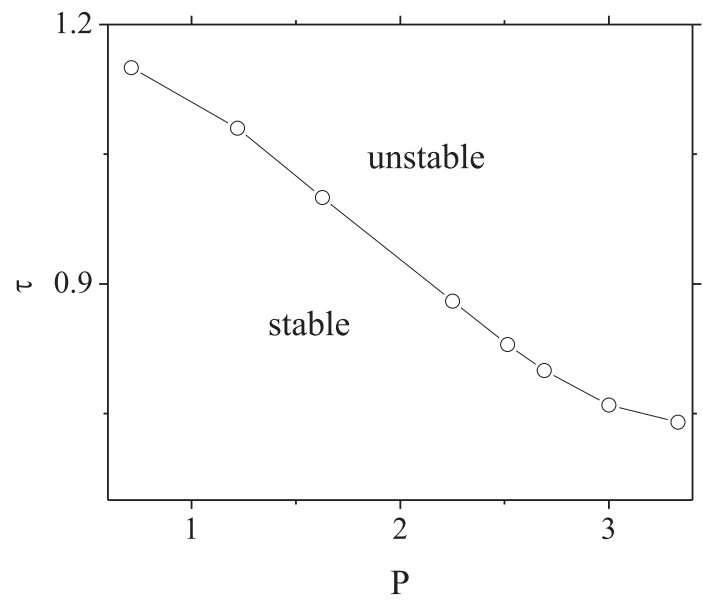

FIG. 7: The border between stable and unstable on-site stationary solitons in the parameter plan of $(\tau, P)$, for coupling parameter $C=0.8$. The instability criterion is $\operatorname{Re}(\mathrm{EV})>10^{-3}$.
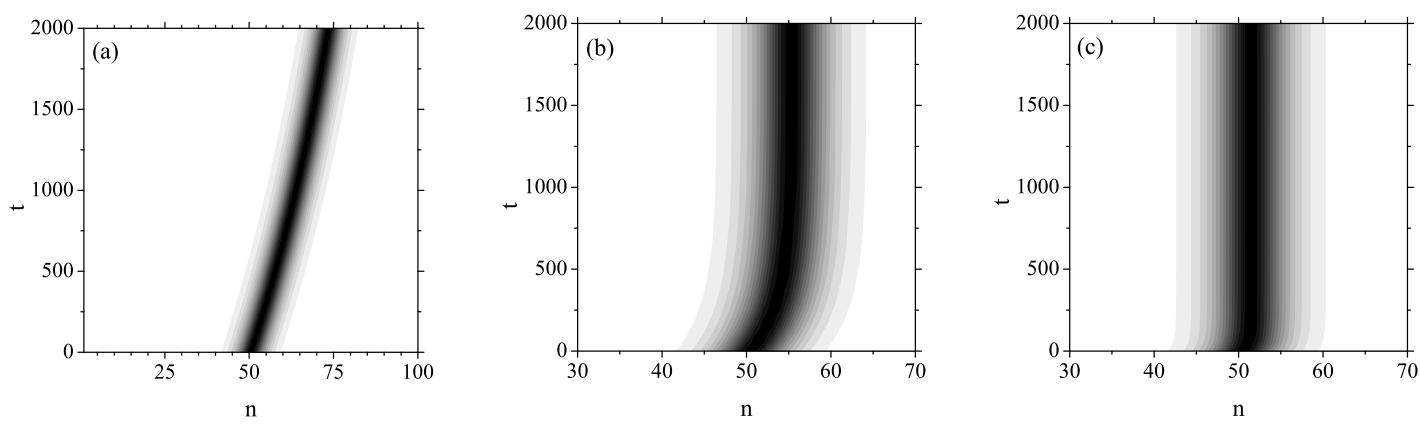

FIG. 8: The evolution of a kicked inter-site soliton with $\mu=-0.12$ and $P=1.22$ in the lattice with time-delayed nonlinearity. The kick's strength is $\alpha=0.01$, and the lattice coupling constant is $C=0.8$. The analysis has shown that this soliton is marginally stable, in its static form, for $\tau=0.01$ and $\tau=0.1$, and unstable for $\tau=0.5$. These values of $\tau$ correspond, respectively, to panels (a), (b), and (c). In the first case (very small $\tau$ ), the kicked soliton keeps moving freely. In the second case (moderately small $\tau$ ), a moving breather is formed, which gradually comes to a halt (b). Large $\tau$ makes the soliton completely immobile (c). 


\section{CONCLUSION}

In this work, we aimed to study the stability and dynamics of unstaggered localized modes in nonlinear lattices with the delayed nonlinear response. The discrete solitons are destroyed when the delay time increases above a certain critical value, in agreement with the observation reported in Ref. [18] that the wave-packet delocalization, implying a breakdown of the self-trapping, is a delay-induced transition.

In the case of the fast nonlinear response (short temporal delay), the exponential-instability growth rate of intersite solitons decreases, in comparison with the instantaneous model. However, full stabilization of the inter-site solitons does not occur. Marginally stable inter-site solitons, which feature a virtually vanishing real instability EV (eigenvalue), and find their on-site counterparts with equal norms, have been found in the case of a small delay time $(\tau)$, as well as for larger $\tau$ near $\mu=0$ (i.e., for broad solitons). Under the action of perturbations, these solitons evolve into breathing localized modes. The marginally stable solitons can be transformed into moving localized modes by the application of a kick.

At large values of $\tau$, all solitons, of both the on-site and inter-site types, are unstable. In direct simulations, they decay into diffractive patterns. In fact, this means that the localized mode spreads out before the nonlinearity commences to act in the slowly responding media. The transition to this instability is indicated by the expansion of the EV spectrum for small perturbations around the discrete soliton, with the emergence of a growing number of complex EVs with significant positive real parts.

The results reported in this work clearly demonstrate that the dynamical properties of localized modes in selffocusing media are essentially affected by the temporal delay of the nonlinearity. These findings complement the recently published study of effects of the time-delayed nonlinear response on the transition to delocalization of wave packets [18]. Altogether, these results provide for an insight into the dynamics of localized patterns in slowly responding media.

A.M. and Lj.H. acknowledge support from the Ministry of Science, Serbia (project 141034). The work of B.A.M. was supported, in a part, by grant No. 149/2006 from the German-Israel Foundation.

[1] A. G. Balanov, N. B. Janson, and Schöll, Phys. Rev. E 71, 016222 (2005).

[2] A. Ahlborn and U. Parlitz, Phys. Rev. E 72, 016206 (2005).

[3] A. Ahlborn and U. Parlitz, Physical Review E 72, 016206 (2005).

[4] Y. Y. Wu, and Y. Q. Wu, International Journal of Automation and Computing 6, 223 (2009).

[5] C. T. Lin, H. W. Nein, and W.-C. Lin, International Journal of Neural Systems 9, 311 (1999).

[6] X. Liao, and J. Wang, Physical Review E 68, 016118 (2005).

[7] A. A. Canabarro, I. M. Gleria, M. L. Lyra, Physica A 342, 234 (2004).

[8] W. Mather, M. R. Bennett, J. Hasty, and L. S. Tsimring, Phys. Rev. Lett. 102, 068105 (2009).

[9] J. Awrejeewicz, Acta Mechanica 77, 111 (1989).

[10] A. Yariv, Y. Xu, R. K. Lee, and A. Scherer, Opt. Lett. 24, 711 (1999).

[11] C. S. Chou and M. F. Shih, J. Opt. A: Pure Appl. Opt. 11, 105204 (2009).

[12] J. K. S. Poon, J. Scheuer, S. Mookherjea, G. T. Paloczi, Y. Y. Huang, and A. Yariv, Opt. Exp. 12, 90 (2004).

[13] Y. C. Kouomou, P. Colet, L. Larger, and N. Gastand, Phys. Rev. Lett. 95, 203903 (2005).

[14] J. K. S. Poon, J. Scheuer, and A. Yariv, J. Opt. Soc. Am. B 21, 1665 (2004).

[15] A. B. Cohen, B. Tavoori, T. E. Murphy, and R. Roy, Phys. Rev. Lett. 101, 154102 (2008).

[16] T. W. Carr, Eur. Phys. J. D 19, 245 (2002).

[17] Y. C. Konomou, P. Colet, L. Larger, and N. Gastaud, Phys. Rev. Lett. 95, 203903 (2005); L. Larger, P.-A. Lacourt, S. Poinsot, and M. Hanna, Phys. Rev. Lett. 95, 043903 (2005).

[18] F. A. B. F. de Moura, I. Gleria, I. F. dos Santos, and M. L. Lyra, Phys. Rev. Lett. 103, 096401 (2009).

[19] P. G. Kevrekidis, editor, The Discrete Nonlinear Schrödinger Equation: Mathematical Analysis, Numerical Computations, and Physical Perspectives (Springer: Berlin and Heidelberg, 2009).

[20] T. Mori, N. Fukuma, and M. Kuwahara, IEEE Trans. Automat. Control 27, 964 (1982).

[21] A. Amann, E. Schöll, and W. Just, Physica A 373, 191 (2007).

[22] K. J. Hout, IMA J. Num. Anal. 17, 17 (1997).

[23] K. Engelborghs, T. Luzyanina, and D. Roose, J. Comp. Appl. Math. 125, 265 (2000).

[24] S. Aubry, Physica D 7, 240 (1983); Yu. S. Kivshar and D. K. Campbell, Phys. Rev. E 48, 3077 (1993).

[25] S. Aubry and T. Cretegny, Physica D 119, 34 (1998); A. Maluckov, Lj. Hadzievski, and M. Stepić, Physica D 216, 95 (2006); G. Gligorić, A. Maluckov, Lj. Hadžievski, and B. A. Malomed, Physical Review A 78, 063615 (2008).

[26] R. M. Corless, G. H. Gonnet, D. E. G. Hare, G. J. Jeffery, and D. E. Knuth, Adv. Comp. Math. 5, 325 (1996).

[27] S. P. Corwin, D. Sarafyan, and S. Thompson, Appl. Num. Math. 24, 319 (1997).

[28] K. Engelborghs, T. Luzyanina, G. Samaey, http://www.cs.kuleuven.ac.be/ koen/delay/ddebiftool.shtml.

[29] L. Salasnich, A. Cetoli, B. A. Malomed, and F. Toigo, Phys. Rev. A 75, 033622 (2007). 